\title{
Reconciling FTIR Spectroscopy with Top-off Operations at the Advanced Light Source
}

\author{
Laetitia Vernoud, Hans A. Bechtel, Ferenc Borondics, \\ and Michael C. Martin
}

Advanced Light Source Division, Lawrence Berkeley National Laboratory,

1 Cyclotron Road, Berkeley, CA 94720, USA

\begin{abstract}
Top-off operations is a quasi-continuous injection mode that increases the flux and brightness of a synchrotron source and improves thermal stability of optical components by maintaining a constant current in the storage ring. Although the increased and constant flux is advantageous for FTIR measurements, the frequent injections (about one every 30 seconds in the ALS case) introduce artifacts into the spectrum by creating spikes in the interferogram data. These spikes are caused by brief beam motion during the injection event. Here, we describe our efforts to minimize the effects of top-off generated interferogram spikes on several FTIR spectrometers. They include using a fast feedback mirror system to correct for beam motion and a gating signal to inhibit interferogram collection during a top-off injection.
\end{abstract}

Keywords: FTIR, synchrotron, infrared radiation, microspectroscopy

PACS: 07.57.-c; 07.85.Qe

\section{INTRODUCTION}

Synchrotron radiation is a bright source of infrared light that can be used with commercial FTIR microscope systems to achieve diffraction limited performance with high signal to noise ratios, thus enabling chemical information to be obtained from small samples. ${ }^{1-3}$ Synchrotron facilities have traditionally operated in decay mode, in which the storage ring is filled to a specified current and then allowed to decay to nominally half the original value before refilling. In decay mode at the ALS, the storage ring was filled to $400 \mathrm{~mA}$ three times a day and the beam would decay to $\sim 200$ $\mathrm{mA}$ in the 8 hours between fills, giving a time-averaged beam current of $250 \mathrm{~mA}$. Although acceptable for most applications, the decaying current and photon flux led to thermal instabilities of beamline optics and was particularly challenging for quantitative measurements.
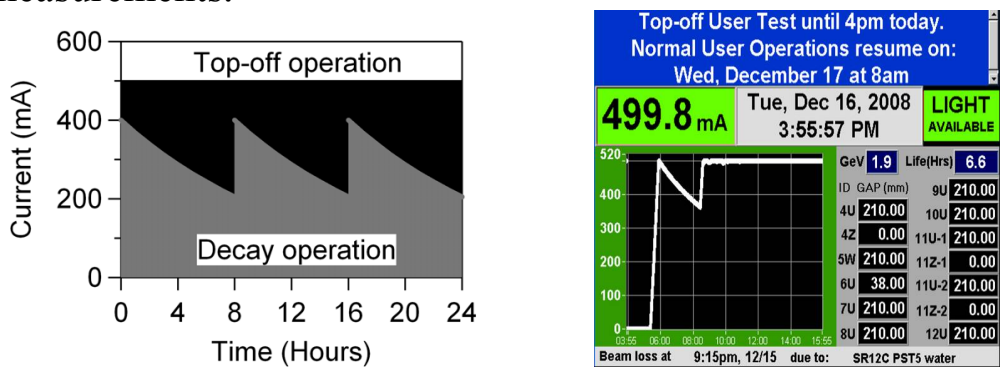

FIGURE 1. Comparison of decay mode and top-off mode at the ALS, and first user top-off tests. 

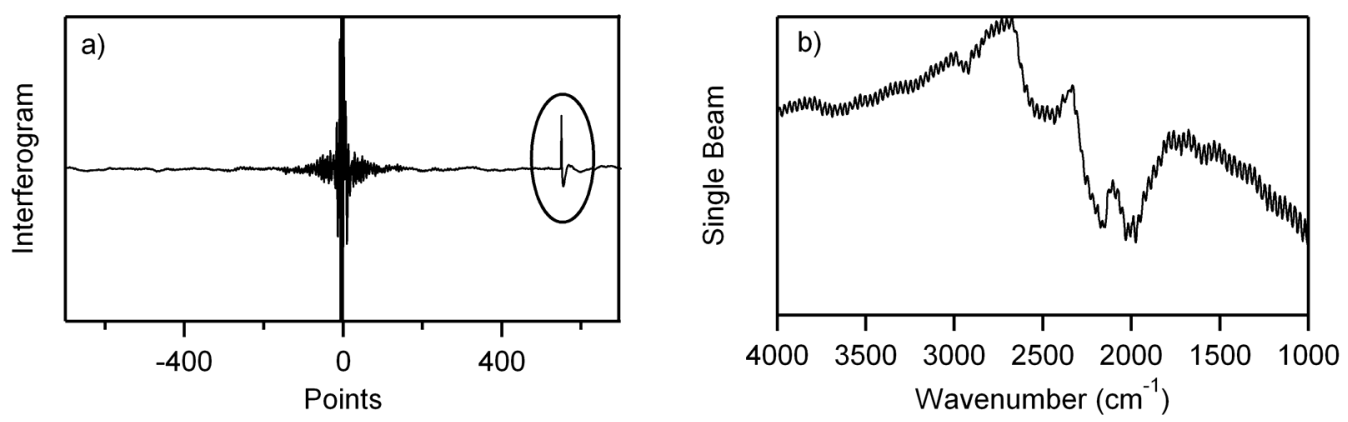

FIGURE 2. (a) Interferogram with a spike caused by an injection during top-off operations. This spike creates an interference pattern in (b) the Fourier transformed and decreases the signal to noise ratio.

In recent years, many synchrotron facilities have been upgrading to top-off operations, a mode where electrons are quasi-continuously injected into the storage ring to maintain a constant current. ${ }^{4}$ As shown in Figure 1, top-off operation has obvious advantages over decay operation in terms of increased flux, brightness, and stability of the synchrotron source. In addition, top-off mode provides continuous light to users because time is not needed to refill the storage ring.

Although top-off operation is superior to decay operation in almost every way, the constant injections create a modest problem for FTIR spectroscopy. During an injection, the orbit of the electron beam is disturbed and the synchrotron light moves a corresponding amount. This fast movement is picked up by the infrared detector and causes a transient spike in the interferogram data, as shown in Fig 2. The spike creates artifacts in the spectrum and decreases the signal-to-noise ratio. Because the injection rate is not synchronized with the scanning mirror of the interferometer, averaging scans reduces the effects of top-off mode injection; however, a method of removing the artifact completely is clearly preferable.

\section{RESULTS}

At the ALS infrared beamlines, we have a fast feedback mirror system to minimize the effects of vibrations and to lock the infrared beam onto the microscope sample. ${ }^{5}$ The feedback system reduces the effects of the top-off mode injections and is particularly good at correcting the longer decay $(\sim 20 \mathrm{~ms})$ of the electron beam back to the normal orbit. The feedback system, however, is not fast enough (rolloff at $\sim 1 \mathrm{kHz}$ ) to correct for the initial transient of the injection event. As a consequence, we have implemented a gating signal to interrupt interferogram collection during an injection event on our Thermo-Nicolet spectrometers. This operation makes use of the Nicolet Start/Stop Accessory, which effectively rejects a scan whenever the gate signal is present, regardless of where it occurs within the scan. The trigger is a signal from the linear accelerator electronics that precedes the injection event by approximately 0.5 seconds. A $30 \mathrm{~ms}$ wide gate is created with a digital delay generator (Stanford Research Systems DG645) approximately $10 \mathrm{~ms}$ before the injection. Because the injection period is short and the time between injections is relatively long, the duty cycle remains high, making the gating system completely transparent to the user. Moreover, accelerator operators may adjust the rate of injection without affecting our 


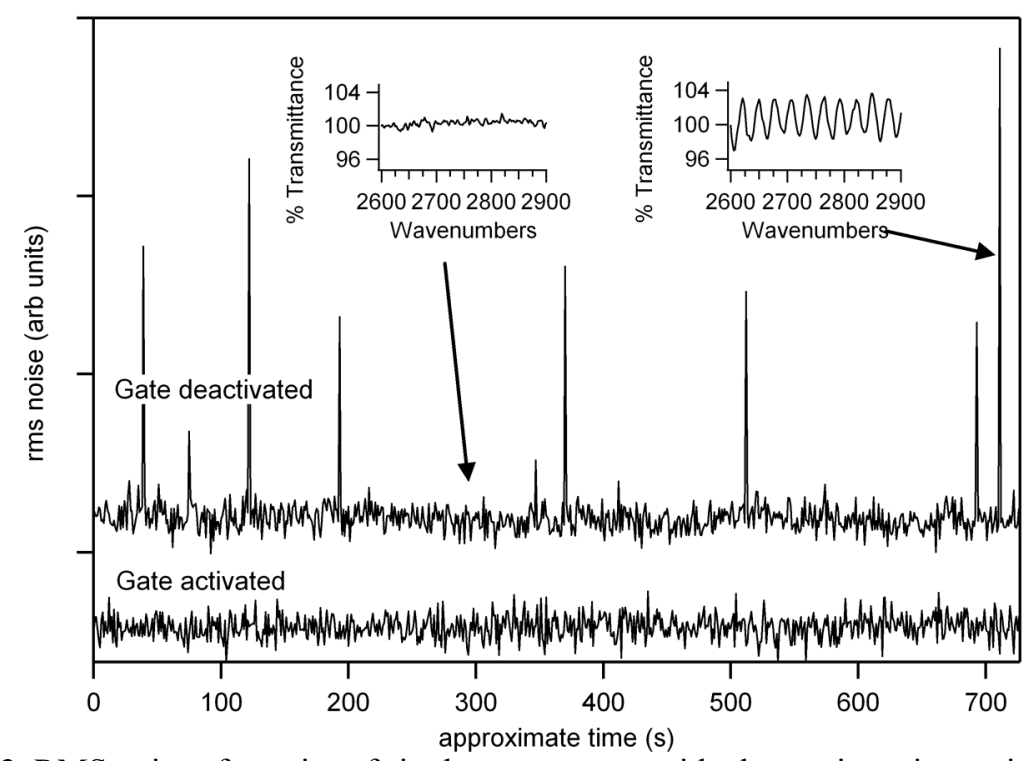

FIGURE 3. RMS noise of a series of single scan spectra with electronic gating activated and deactivated. The baseline for the deactivated series has been offset for clarity.

data collection. Figure 3 shows the improved rms noise for single scans collected in series when the gate is activated.

The gating method works well as long as the interferometer scan is shorter than the time between injection events. At standard mirror velocities, we find that a frequency resolution of $0.125 \mathrm{~cm}^{-1}$ is achievable without considerable reduction in the duty cycle. For higher resolution measurements, however, an interferometer scan can take longer than the time between injection events. In particular, high resolution instruments, such as the Bruker IFS 125HR, can take greater than 6 minutes for a single scan at $0.001 \mathrm{~cm}^{-1}$ resolution. In this case, we would expect $\sim 12$ top-off artifacts will be in each interferogram. Clearly, another approach is needed for these measurements. We are investigating analog circuits between the detector and analog to digital converter as well as software corrections to affected interferograms.

\section{ACKNOWLEDGMENTS}

The Advanced Light Source is supported by the Director, Office of Science, Office of Basic Energy Sciences, of the U.S. Department of Energy under Contract No. DE-AC02-05CH11231.

\section{REFERENCES}

1 J. A. Reffner, P. A. Martoglio, and G. P. Williams, Rev. Sci. Instrum. 66, 1298 (1995).

2 G. L. Carr, Rev. Sci. Instrum. 72, 1613 (2001).

3 H.-Y. N. Holman and M. C. Martin, Adv. Agron. 90, 79 (2006).

4 C. Steier, et al., 2007 IEEE Particle Accelerator Conference, 1197 (2007).

5 T. Scarvie, N. Andresen, K. Baptiste, J. Byrd, M. Chin, M. C. Martin, W. R. McKinney, and C. Steier, Infrared Phys. Techn. 45, 403 (2004). 